\title{
Lattices with many congruences are planar
}

\author{
Gábor Czédli
}

Dedicated to the memory of Ivan Rival.

\begin{abstract}
Let $L$ be an $n$-element finite lattice. We prove that if $L$ has more than $2^{n-5}$ congruences, then $L$ is planar. This result is sharp, since for each natural number $n \geq 8$, there exists a non-planar lattice with exactly $2^{n-5}$ congruences.
\end{abstract}

Mathematics Subject Classification. 06B10.

Keywords. Planar lattice, Lattice congruence, Congruence lattice.

\section{Aim and introduction}

Our goal is to prove the following statement.

Theorem 1.1. Let $L$ be an $n$-element finite lattice. If $L$ has more than $2^{n-5}$ congruences, then it is a planar lattice.

In order to point out that this result is sharp, we will also prove the following easy remark. An $n$-element finite lattice $L$ is dismantlable if there is a sequence $L_{1} \subset L_{2} \subset \cdots \subset L_{n}=L$ of its sublattices such that $\left|L_{i}\right|=i$ for every $i \in\{1, \ldots, n\}$; see Rival [13]. We know from Kelly and Rival [9] that every finite planar lattice is dismantlable.

Remark 1.2. For each natural number $n \geq 8$, there exists an $n$-element nondismantlable lattice $L(n)$ with exactly $2^{n-5}$ congruences; this $L(n)$ is nonplanar.

We know from Freese [5] that an $n$-element lattice $L$ has at most $2^{n-1}=$ $16 \cdot 2^{n-5}$ congruences. In other words, denoting the lattice of congruences of

Presented by J.B. Nation.

This research was supported by the National Research, Development and Innovation Fund of Hungary under the KH 126581 funding scheme. 
$L$ by $\operatorname{Con}(L)$, we have that $|\operatorname{Con}(L)| \leq 2^{n-1}$. For $n \geq 5$, the second largest number of the set

$$
\operatorname{ConSizes}(n):=\{|\operatorname{Con}(L)|: L \text { is a lattice with }|L|=n\}
$$

is $8 \cdot 2^{n-5}$ by Czédli [3], while Kulin and Mureşan [10] proved that the third, fourth, and fifth largest numbers of $\operatorname{ConSizes}(n)$ are $5 \cdot 2^{n-5}, 4 \cdot 2^{n-5}$, and $\frac{7}{2} \cdot 2^{n-5}$, respectively. Since both [3] and Kulin and Mureşan [10] described the lattices witnessing these numbers, it follows from these two papers that $|\operatorname{Con}(L)| \geq \frac{7}{2} \cdot 2^{n-5}$ implies the planarity of $L$. So, [3], [10], and even their precursor, Mureşan [11] have naturally lead to the conjecture that if an $n$ element lattice $L$ has many congruences with respect to $n$, then $L$ is necessarily planar. However, the present paper needs a technique different from Kulin and Mureşan [10], because a [10]-like description of the lattices witnessing the sixth, seventh, eighth, $\ldots, k$-th largest numbers in $\operatorname{ConSizes}(n)$ seems to be hard to find and prove; we do not even know how large $k$ is. Fortunately, we can rely on the powerful characterization of planar lattices given by Kelly and Rival [9].

Note that although an $n$-element finite lattice with "many" (that is, more than $2^{n-5}$ ) congruences is necessarily planar by Theorem 1.1 , an $n$ element planar lattice may have only very few congruences even for large $n$. For example, the $n$-element modular lattice of length 2 , denoted usually by $M_{n-2}$, has only two congruences if $n \geq 5$. On the other hand, we know, say, from Kulin and Mureşan [10] that there are a lot of lattices $L$ with many congruences, whereby a lot of lattices belong to the scope of Theorem 1.1.

\section{Outline and prerequisites}

Section 2 recalls some known facts from the literature and, based on these facts, proves Remark 1.2 in three lines. The rest of the paper is devoted to the proof of Theorem 1.1.

Due to Section 2, the reader is assumed to have only little familiarity with lattices. Apart from some figures from Kelly and Rival [9], which should be at hand while reading, the present paper is more or less self-contained modulo the above-mentioned familiarity. Note that [9] is an open access paper at the time of this writing; see http://dx.doi.org/10.4153/CJM-1975-074-0.

\section{Some known facts about lattices and their congruences}

In the whole paper, all lattices are assumed to be finite even if this is not repeated all the time. For a finite lattice $L$, the set of nonzero join-irreducible elements, that of nonunit meet-irreducible elements, and that of doubly irreducible (neither 0 , not 1 ) elements will be denoted by $\mathrm{J}(L), \mathrm{M}(L)$, and $\operatorname{Irr}(L)=$ $\mathrm{J}(L) \cap \mathrm{M}(L)$, respectively. For $a \in \mathrm{J}(L)$ and $b \in \mathrm{M}(L)$, the unique lower cover of $a$ and the unique (upper) cover of $b$ will be denoted by $a^{-}$and $b^{+}$, respectively. For $a, b \in L$, let $\operatorname{con}(a, b)$ stand for the smallest congruence of $L$ such that $\langle a, b\rangle \in \operatorname{con}(a, b)$. For $x, y \in \mathrm{J}(L)$, let $x \equiv_{\text {con }} y$ mean that 
$\operatorname{con}\left(x^{-}, x\right)=\operatorname{con}\left(y^{-}, y\right)$. Then $\equiv_{\text {con }}$ is an equivalence relation on $\mathrm{J}(L)$, and the corresponding quotient set will be denoted by

$$
\mathrm{Q}(L):=\mathrm{J}(L) / \equiv_{\text {con }} \text {. }
$$

As an obvious consequence of Freese, Ježek and Nation [6, Theorem 2.35] or Nation [12, Corollary to Theorem 10.5], for every finite lattice $L$,

$$
|\operatorname{Con}(L)| \leq 2^{|\mathrm{Q}(L)|} \leq 2^{|\mathrm{J}(L)|} ;
$$

more explanation of this fact and (2.3) below will be given later. The situation simplifies for distributive lattices; it is well known that

if $L$ is a finite distributive lattice, then $|\operatorname{Con}(L)|=2^{|\mathrm{J}(L)|}$.

Next, having no explicit reference at hand, we give a possible way how to extract (2.2) and (2.3) from the literature; the reader may skip over these details. A quasiordered set is a structure $\langle A ; \leq\rangle$ where $\leq$ is a quasiordering, that is, a reflexive and symmetric relation on $A$. For example, if we let $a \leq_{\text {con }} b$ mean $\operatorname{con}\left(a^{-}, a\right) \leq \operatorname{con}\left(b^{-}, b\right)$, then $\left\langle\mathrm{J}(L) ; \leq_{\text {con }}\right\rangle$ is a quasiordered set. A subset $X$ of $\langle A ; \leq\rangle$ is hereditary, if $(\forall x \in X)(\forall y \in A)(y \leq x \Rightarrow y \in X)$. The set of all hereditary subsets of $\langle A ; \leq\rangle$ with respect to set inclusion forms a lattice $\operatorname{Hered}(\langle A ; \leq\rangle)$. Freese, Ježek and Nation [6, Theorem 2.35] can be reworded as $\langle\operatorname{Con}(L) ; \subseteq\rangle \cong \operatorname{Hered}\left(\left\langle\mathrm{J}(L) ; \leq_{\text {con }}\right\rangle\right)$. To recall this theorem in a form closer to $\left[6\right.$, Theorem 2.35], for the $\equiv_{\text {con-blocks of } a, b \in \mathrm{J}(L) \text {, we define the meaning }}$ of $a / \equiv_{\text {con }} \leq_{\text {con }} b / \equiv_{\text {con }}$ as $a \leq_{\text {con }} b$. In this way, we obtain a poset (partially ordered set) $\left\langle\mathrm{Q}(L) ; \leq_{\text {con }} / \equiv_{\text {con }}\right\rangle$. With this notation, the original form of $[6$, Theorem 2.35] states that $\operatorname{Con}(L) \cong \operatorname{Hered}\left(\mathrm{Q}(L) ; \leq_{\text {con }} / \equiv_{\text {con }}\right)$, which implies (2.2).

Since $\equiv_{\text {con }}$ will play an important role later, recall that for intervals $[a, b]$ and $[c, d]$ in a lattice $L,[a, b]$ transposes up to $[c, d]$ if $b \wedge c=a$ and $b \vee c=d$. This relation between the two intervals will be denoted by $[a, b] \nearrow[c, d]$. We say that $[a, b]$ transposes down to $[c, d]$, in notation $[a, b] \searrow[c, d]$ if $[c, d] \nearrow[a, b]$. We call $[a, b]$ and $[c, d]$ transposed intervals if $[a, b] \searrow[c, d]$ or $[a, b] \nearrow[c, d]$. It is well known and easy to see that

if $[a, b]$ and $[c, d]$ are transposed intervals, then $\operatorname{con}(a, b)=\operatorname{con}(c, d)$.

Note that $\operatorname{Con}(L)$ in $(2.3)$ is a Boolean lattice. Even more is true: $\operatorname{Con}(L)$ is Boolean for every finite modular lattice; this follows from the characterization of lattice congruences given in Dilworth [4] and it is explicit in the monograph Crawley and Dilworth [2, 10.3 combined with 10.7]. Next, let $L$ be a finite distributive lattice, and pick a maximal chain $0 \prec a_{1} \prec \cdots \prec a_{t}=1$ in $L$. Here $t$ is the length of $L$, and it is well known that $t=|\mathrm{J}(L)|$; see, for example, Grätzer [7, Corollary 112 in page 114]. If $\operatorname{con}\left(a_{i-1}, a_{i}\right)=\operatorname{con}\left(a_{j-1}, a_{j}\right)$, then it follows from Crawley and Dilworth [2, 10.2 and 10.3] or from Grätzer [8] that there is a sequence of prime intervals (edges in the diagram) from $\left[a_{i-1}, a_{i}\right]$ to $\left[a_{j-1}, a_{j}\right]$ such that any two neighboring intervals in this sequence are transposed. (We know from Crawley and Dilworth $[2,10.4]$ that there exists such a sequence even of length two, but we do not need this fact.) 
In the terminology of Adaricheva and Czédli [1], the existence of the above-mentioned sequence means that $\left[a_{i-1}, a_{i}\right]$ and $\left[a_{j-1}, a_{j}\right]$ belong to the same trajectory. Since no two distinct comparable prime intervals of $L$ can belong to the same trajectory by $[1$, Proposition 6.1$]$, it follows that $i=j$. Hence, the congruences $\operatorname{con}\left(a_{i-1}, a_{i}\right), i \in\{1, \ldots, t\}$, are pairwise distinct. It is well known that a prime interval in a finite lattice generates a joinirreducible congruence; see, for example, Grätzer [7, page 213]. Hence, the $\operatorname{con}\left(a_{i-1}, a_{i}\right), i \in\{1, \ldots, t\}$, are atoms in $\operatorname{Con}(L)$ since $\operatorname{Con}(L)$ is Boolean. Clearly, $\bigvee_{i=1}^{t} \operatorname{con}\left(a_{i-1}, a_{i}\right)$ is $1_{\operatorname{Con}(L)}$, which implies that $|\operatorname{Con}(L)|=2^{t}$. This proves $(2.3)$ since $t=|\mathrm{J}(L)|$.

Next, a lattice is called planar if it is finite and has a Hasse-diagram that is a planar representation of a graph in the usual sense that any two edges can intersect only at a vertex. For lattices $K$ and $L$, we say that $L$ contains $K$ as a subposet if there exists an injective map $\varphi: K \rightarrow L$ such that, for all $x, y \in K$, we have $x \leq y$ in $K$ if and only if $\varphi(x) \leq \varphi(y)$ in $L$. If, in addition, $K \subseteq L$ and the inclusion map $\iota: K \rightarrow L$, defined by $x \mapsto x$, has the same property as $\varphi$ above, then the lattice $K$ is a subposet of $L$. If $K$ is only a poset but need not be a lattice, then the same condition defines that $K$ is (isomorphic to) a subposet of $L$. Let $\mathbb{N}_{0}$ and $\mathbb{N}^{+}$denote the set $\{0,1,2, \ldots\}$ of nonnegative integers and the set $\{1,2,3, \ldots\}$ of positive integers, respectively. In their fundamental paper on planar lattices, Kelly and Rival [9] gave a set

$$
\mathcal{L}_{\mathrm{KR}}=\left\{A_{n}, E_{n}, F_{n}, G_{n}, H_{n}: n \in \mathbb{N}_{0}\right\} \cup\{B, C, D\}
$$

of finite lattices such that the following statement holds.

Proposition 2.1 (A part of Kelly and Rival [9, Theorem 1]). A finite lattice $L$ is planar if and only if neither $L$, nor its dual contains some lattice of $\mathcal{L}_{K R}$ as a subposet.

Note that the lattices $A_{n}, F_{n}, G_{n}$, and $H_{n}$ are selfdual. Note also that Kelly and Rival [9] proved the minimality of $\mathcal{L}_{\mathrm{KR}}$, but we do not need this fact.

Next, we prove Remark 1.2. The ordinal sum of lattices $L^{\prime}$ and $L^{\prime \prime}$ is their disjoint union $L^{\prime} \dot{\cup} L^{\prime \prime}$ such that for $x, y \in L^{\prime} \dot{\cup} L^{\prime \prime}$, we have that $x \leq y$ if and only if $x \leq_{L^{\prime}} y$, or $x \leq_{L^{\prime \prime}} y$, or $x \in L^{\prime}$ and $y \in L^{\prime \prime}$.

Proof of Remark 1.2. Let $L(8)$ be the eight-element Boolean lattice. Also, for $n>8$, let $L(n)$ be the ordinal sum of $L(8)$ and an $(n-8)$-element chain. Since $\left|\mathrm{J}\left(L_{n}\right)\right|=n-5$, Remark 1.2 follows from (2.3).

Note that $L(n)$ above occurs also in page 93 of Rival [13].

\section{A lemma on subposets that are lattices}

While $\mathcal{L}_{\mathrm{KR}}$ consists of lattices, they appear in Proposition 2.1 as subposets. This fact causes some difficulties in proving our theorem; this section serves as 
a preparation to overcome them. The set of join-reducible elements of a lattice $L$ will be denoted by $\operatorname{JRed}(L)$. Note that

$$
\operatorname{JRed}(L)=L \backslash(\{0\} \cup \mathrm{J}(L))=\{a \vee b: a, b \in L \text { and } a \| b\},
$$

where $\|$ stands for incomparability, that is, $a \| b$ is the conjunction of $a \not \leq b$ and $b \not \leq a$. Similarly, $\operatorname{MRed}(L)=L \backslash(\{1\} \cup \mathrm{M}(L))$ denotes the set of meet-reducible elements of $L$.

Lemma 3.1. Let $L$ and $K$ be finite lattices such that $K$ is a subposet of $L$. Then the following four statements and their duals hold.

(i) If $a_{1}, \ldots, a_{t} \in K$ and $t \in \mathbb{N}^{+}$, then $a_{1} \vee_{L} \cdots \vee_{L} a_{t} \leq a_{1} \vee_{K} \cdots \vee_{K} a_{t}$.

(ii) If $t, s \in \mathbb{N}^{+}, a_{1}, \ldots, a_{t}, b_{1}, \ldots, b_{s} \in K$, and $a_{1} \vee_{K} \cdots \vee_{K} a_{t}$ is distinct from $b_{1} \vee_{K} \cdots \vee_{K} b_{s}$, then $a_{1} \vee_{L} \cdots \vee_{L} a_{t} \neq b_{1} \vee_{L} \cdots \vee_{L} b_{s}$.

(iii) $|\operatorname{JRed}(L)| \geq|\operatorname{JRed}(K)|$ and, dually, $|\operatorname{MRed}(L)| \geq|\operatorname{MRed}(K)|$.

(iv) If $|\operatorname{JRed}(L)|=|\operatorname{JRed}(K)|, u_{1}, u_{2}, v_{1}, v_{2} \in K, u_{1}\left\|u_{2}, v_{1}\right\| v_{2}$, and $u_{1} \vee_{K} u_{2}=v_{1} \vee_{K} v_{2}$, then $u_{1} \vee_{L} u_{2}=v_{1} \vee_{L} v_{2}$.

Note that, according to (ii) and (iv), the distinctness of joins is generally preserved when passing from $K$ to $L$, but equalities are preserved only under additional assumptions. The dual of a condition or statement $(\mathrm{X})$ will often be denoted by $(\mathrm{X})^{\mathrm{d}}$; for example, the dual of Lemma 3.1(i) is denoted by Lemma $3.1(\mathrm{i})^{\mathrm{d}}$ or simply by $3.1(\mathrm{i})^{\mathrm{d}}$.

Proof of Lemma 3.1. Part (i) is a trivial consequence of the concept of joins as least upper bounds.

In order to prove (ii), assume that $a_{1} \vee_{L} \cdots \vee_{L} a_{t}=b_{1} \vee_{L} \cdots \vee_{L} b_{s}$. Part (i) gives that $a_{i} \leq_{L} \quad a_{1} \vee_{L} \cdots \vee_{L} a_{t}=b_{1} \vee_{L} \cdots \vee_{L} b_{s} \leq_{L} \quad b_{1} \vee_{K} \cdots \vee_{K} b_{s}$, for all $i \in\{1, \ldots, t\}$. Since $K$ is a subposet of $L, a_{i} \leq_{K} b_{1} \vee_{K} \cdots \vee_{K} b_{s}$. But $i \in$ $\{1, \ldots, t\}$ is arbitrary, whereby $a_{1} \vee_{K} \cdots \vee_{K} a_{t} \leq_{K} b_{1} \vee_{K} \cdots \vee_{K} b_{s}$. We have equality here, since the converse inequality follows in the same way. Thus, we conclude (ii) by contraposition.

Next, let $\left\{c_{1}, \ldots, c_{t}\right\}$ be a repetition-free list of $\operatorname{JRed}(K)$. For each $i$ in $\{1, \ldots, t\}$, pick $a_{i}, b_{i} \in K$ such that $a_{i} \| b_{i}$ and $c_{i}=a_{i} \vee_{K} b_{i}$. That is,

$$
\operatorname{JRed}(K)=\left\{c_{1}=a_{1} \vee_{K} b_{1}, \ldots, c_{t}=a_{t} \vee_{K} b_{t}\right\}
$$

Since $a_{i} \| b_{i}$ holds also in $L$,

$$
\left\{a_{1} \vee_{L} b_{1}, \ldots, a_{t} \vee_{L} b_{t}\right\} \subseteq \operatorname{JRed}(L) .
$$

The elements listed in (3.3) are pairwise distinct by part (ii). Therefore, $|\operatorname{JRed}(K)|=t \leq|\operatorname{JRed}(L)|$, proving part (iii).

Finally, to prove part (iv), we assume its premise, and we let $t:=$ $|\operatorname{JRed}(K)|=|\operatorname{JRed}(L)|$. Choose $c_{i}, a_{i}, b_{i} \in K$ as in (3.2). Since $t=|\operatorname{JRed}(L)|$, part (ii) and (3.3) give that

$$
\operatorname{JRed}(L)=\left\{a_{1} \vee_{L} b_{1}, \ldots, a_{t} \vee_{L} b_{t}\right\} .
$$

As a part of the premise of (iv), $u_{1} \| u_{2}$ has been assumed. Hence, $u_{1} \vee_{K} u_{2} \in$ $\operatorname{JRed}(K)$, and so (3.2) yields a unique subscript $i \in\{1, \ldots, t\}$ such that $c_{i}=u_{1} \vee_{K} u_{2}=v_{1} \vee_{K} v_{2}$. Since $c_{1}, \ldots, c_{t}$ is a repetition-free list of the elements of $\operatorname{JRed}(K)$, we have that $u_{1} \vee_{K} u_{2} \neq c_{j}=a_{j} \vee_{K} b_{j}$ for every $j \in$ 

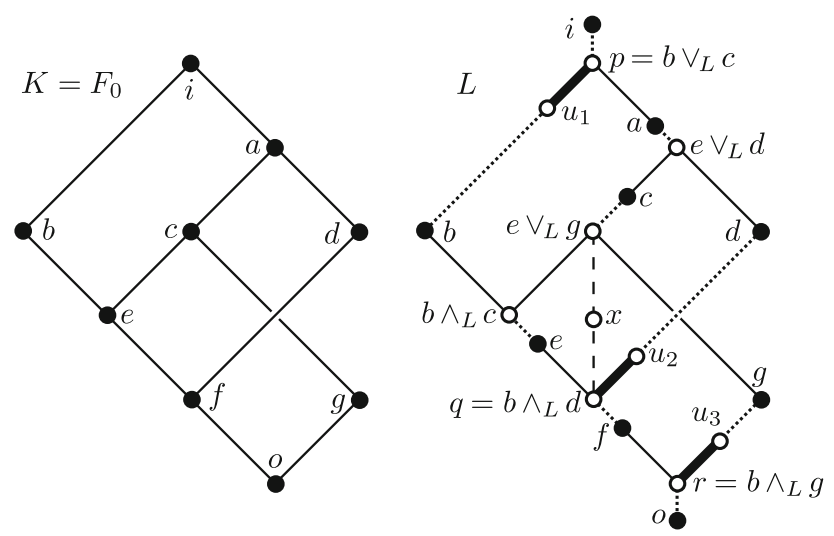

FiguRE $1 . K=F_{0}$ and an example for $L$ containing $K$ as a subposet
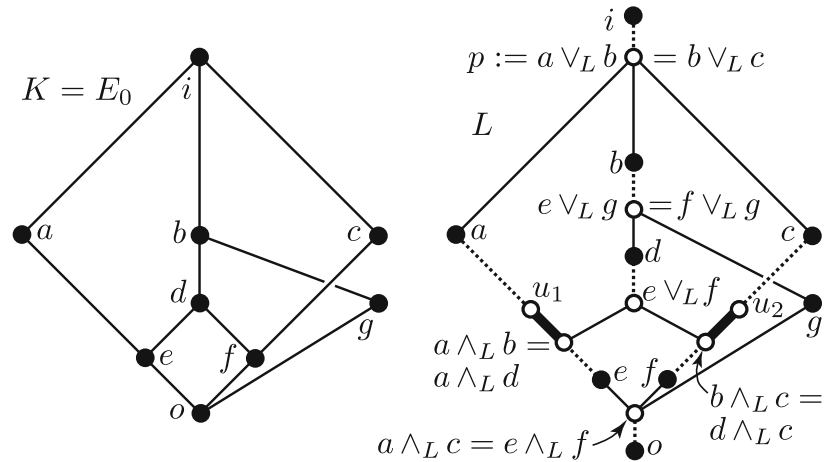

Figure 2. $K=E_{0}$ and an example for $L$ containing $K$ as a subposet

$\{1, \ldots, t\} \backslash\{i\}$. So, for all $j \neq i$, part (ii) gives that $u_{1} \vee_{L} u_{2} \neq a_{j} \vee_{L} b_{j}$ But $u_{1} \vee_{L} u_{2} \in \operatorname{JRed}(L)$, whence (3.4) gives that $u_{1} \vee_{L} u_{2}=a_{i} \vee_{L} b_{i}$. Since the equality $v_{1} \vee_{L} v_{2}=a_{i} \vee_{L} b_{i}$ follows in the same way, we conclude that $u_{1} \vee_{L} u_{2}=v_{1} \vee_{L} v_{2}$, as required. This yields part (iv) and completes the proof of Lemma 3.1.

Note that $a_{i} \vee_{L} b_{i}$ in the proof above can be distinct from $c_{i}$; this will be exemplified by Figures 1 and 2 .

\section{The rest of the proof}

In this section, to ease our terminology, let us agree on the following convention. We say that a finite lattice $L$ has many congruences if $|\operatorname{Con}(L)|>2^{|L|-5}$. Otherwise, if $|\operatorname{Con}(L)| \leq 2^{|L|-5}$, then we say that $L$ has few congruences.

Lemma 4.1. For every finite lattice $L$, the following two assertions holds.

(i) If $|\operatorname{JRed}(L)| \geq 4$ or $|\operatorname{MRed}(L)| \geq 4$, then $L$ has few congruences. 
(ii) If $|\operatorname{JRed}(L)|=3$ and there are $p, q \in \mathrm{J}(L)$ such that $p \neq q$ and $\operatorname{con}\left(p^{-}, p\right)$ $=\operatorname{con}\left(q^{-}, q\right)$, then $L$ has few congruences.

Proof. Let $n:=|L|$. If $|\operatorname{JRed}(L)| \geq 4$, then (3.1) leads to $|\mathrm{J}(L)| \leq n-5$, and it follows by (2.2) that $L$ has few congruences. By duality, this proves part (i). Under the assumptions of (ii), $p \equiv_{\text {con }} q$, and we obtain from (2.1) that $|\mathrm{Q}(L)| \leq|\mathrm{J}(L)|-1=n-4-1=n-5$, and (2.2) implies again that $L$ has few congruences. This proves the lemma.

Lemma 4.2. Let $K:=F_{0} \in \mathcal{L}_{K R}$, see on the left in Figure 1 . If $K$ is a subposet of a finite lattice $L$, then $L$ has few congruences.

Proof. Label the elements of $K=F_{0}$ as shown in Figure 1. A possible $L$ is given on the right in Figure 1; the elements of $K$ are black-filled. The diagram of $L$ is understood as follows: for $y_{1}, y_{2} \in L$, a thick solid edge, a thin solid edge, and a thin dotted edge ascending from $y_{1}$ to $y_{2}$ mean that, in the general case, we know that $y_{1} \prec y_{2}, y_{1}<y_{2}$, and $y_{1} \leq y_{2}$, respectively. In a concrete situation, further relations can be fulfilled; for example, a thin dotted edge can happen to denote that $y_{1}=y_{2}$. The two dashed edges and the element $x$ as well as similar edges and elements can be present but they can also be missing. Note that $y_{1} \leq y_{2}$ is understood as $y_{1} \leq_{L} y_{2}$; for $y_{1}, y_{2} \in K$, this is the same as $y_{1} \leq_{K} y_{2}$ since $K$ is a subposet of $L$. Since $L$ in the figure carries a lot of information on the general case, the reader may choose to inspect Figure 1 instead of checking some of our computations that will come later. Note also that the convention above applies only for $L$; for $K$, every edge in the left of Figure 1 stands for covering.

Clearly, $|\operatorname{JRed}(K)|=|\operatorname{MRed}(K)|=3$. Hence, Lemma 3.1(iii) gives that $|\operatorname{JRed}(L)| \geq 3$ and $|\operatorname{MRed}(L)| \geq 3$. We can assume that none of $|\operatorname{JRed}(L)| \geq 4$ and $|\operatorname{MRed}(L)| \geq 4$ holds, because otherwise Lemma 4.1(i) would immediately complete the proof. Hence,

$$
|\operatorname{JRed}(L)|=3 \quad \text { and }|\operatorname{MRed}(L)|=3 .
$$

Since $|\operatorname{JRed}(K)|=|\operatorname{MRed}(K)|=3$ holds also for $K=E_{0}$, to be given later in Figure 2, note at this point that (4.1) will be valid in the proof of Lemma 4.3. Let $p:=b \vee_{L} c \in L$, and let $u_{1} \in L$ be a lower cover of $p$ in the interval $[b, p]_{L}$. Also, let $q:=b \wedge_{L} d$ and let $u_{2} \in[q, d]_{L}$ be a cover of $q$. Finally, let $r:=b \wedge_{L} g$, and let $u_{3} \in[r, g]_{L}$ be a cover of $r$. Since we have formed the joins and the meets of incomparable elements in $L$ such that the corresponding joins are pairwise distinct in $K$ and the same holds for the meets, (4.1) and Lemma 3.1 imply that

$$
\operatorname{JRed}(L)=\left\{p, e \vee_{L} d, e \vee_{L} g,\right\} \quad \text { and } \operatorname{MRed}(L)=\left\{q, r, b \wedge_{L} c\right\} .
$$

In order to justify some features of Figure 1, note that (4.2) implies easily that $a<p, c<e \vee_{L} d, q<e$, and $r<f$, but we will not use these inequalities. For example, we obtain $a<p$ as follows. Since $b \not \leq a$, we have that $p \not \leq a$. For the sake of contradiction, suppose that $a \| p$. Then $e \vee_{L} g \leq b \vee_{L} c=p<a \vee_{L} p$ and, by Lemma 3.1(i), $e \vee_{L} d \leq e \vee_{K} d=a<a \vee_{L} p$, whereby (4.2) gives that 
$a<a \vee_{L} p$ is strictly larger than every element of $\operatorname{JRed}(L)$, which contradicts $a \vee_{L} p \in \operatorname{JRed}(L)$. Hence, $a<p$.

Since $u_{1} \prec_{L} p, u_{1} \neq p$. If we had that $u_{1}=e \vee_{L} d$, then

$$
b \leq u_{1}=e \vee_{L} d \stackrel{3.1(\mathrm{i})}{\leq} e \vee_{K} d=a
$$

would contradict $b \varliminf_{K} a$. Replacing $\langle d, a\rangle$ by $\langle g, c\rangle$, we obtain similarly that $u_{1} \neq e \vee_{L} g$. Hence, (4.2) gives that $u_{1} \in \mathrm{J}(L)$. If we had that $u_{2}=p$, then $b \leq p=u_{2} \leq d$ would be a contradiction. Similarly, $u_{2}=e \vee_{L} d$ would lead to $e \leq e \vee_{L} d=u_{2} \leq d$ while $u_{2}=e \vee_{L} g$ again to $e \leq e \vee_{L} g=u_{2} \leq d$, which are contradictions. Hence, $u_{2} \notin \operatorname{JRed}(L)$ and so $0_{L} \leq q \prec_{L} u_{2}$ gives that $u_{2} \in \mathrm{J}(L)$. We have that $u_{3} \neq p$, because otherwise $b \leq p=u_{3} \leq g$ would be a contradiction. Similarly, $u_{3}=e \vee_{L} d$ and $u_{3}=e \vee_{L} g$ would lead to the contradictions $e \leq e \vee_{L} d=u_{3} \leq g$ and $e \leq e \vee_{L} g=u_{3} \leq g$, respectively. So, $u_{3} \notin \operatorname{JRed}(L)$ by (4.2). Since $r \prec_{L} u_{3}$ excludes that $u_{3}=0$, we obtain that $u_{3} \in \mathrm{J}(L)$. Since $u_{3}=u_{2}$ would lead to

$$
f=b \wedge_{K} d \stackrel{3.1(\mathrm{i})^{\mathrm{d}}}{\leq} b \wedge_{L} d=q \leq u_{2}=u_{3} \leq g,
$$

which is a contradiction, we have that

$$
u_{1}, u_{2}, u_{3} \in \mathrm{J}(L) \text { and } u_{2} \neq u_{3} .
$$

Next, we claim that

$$
\left[q, u_{2}\right] \nearrow\left[u_{1}, p\right] \text { and }\left[u_{1}, p\right] \searrow\left[r, u_{3}\right] .
$$

Since $b \not \leq a, b \not \leq c$, and $b \not \leq d$, none of $e \vee_{L} d, e \vee_{L} g$, and $u_{2}$ belongs to $[b, i]_{L}$. In particular, we obtain from $u_{1} \prec p$ and (4.2) that

$$
\left[b, u_{1}\right]_{L} \subseteq \mathrm{J}(L) \text { and } b \not u_{2} \text {. }
$$

Suppose, for a contradiction, that $u_{2} \leq u_{1}$, and pick a maximal chain in the interval $\left[u_{2}, u_{1}\right]$. So we pick a lower cover of $u_{1}$, then a lower cover of the previous lower cover, etc., and it follows from (4.6) that this chain contains $b$. Hence, $u_{2} \leq b$, and we obtain that $q \prec_{L} u_{2} \leq b \wedge_{L} d=q$, a contradiction. Hence, $u_{2} \not u_{1}$. This means that $u_{1} \wedge_{L} u_{2}<u_{2}$. But $q \leq b \leq u_{1}$, so we have that $q \leq u_{1} \wedge u_{2}<u_{2}$. Since $q \prec_{L} u_{2}$, we obtain that $u_{1} \wedge_{L} u_{2}=q$. Similarly, $u_{2} \leq d \leq p$ and $u_{2} \not \leq u_{1}$ give that $u_{1}<u_{1} \vee_{L} u_{2} \leq p$, whereby $u_{1} \prec_{L} p$ yields that $u_{1} \vee_{L} u_{2}=p$. The last two equalities imply the first half of (4.5). The second half follows basically in the same way, so we give less details. Based on (4.6), $u_{3} \leq u_{1}$ would lead to $u_{3} \leq b$ and $r \prec_{L} u_{3} \leq b \wedge_{L} g=r$, whence $u_{3} \not u_{1}$. Since $u_{3} \leq g \leq c \leq b \vee_{L} c=p$ and $r=b \wedge_{L} g \leq b \leq u_{1}$, we obtain that $u_{1}<u_{1} \vee_{L} u_{3} \leq p$ and $r \leq u_{1} \wedge_{L} u_{3}<u_{3}$. Hence the covering relations $u_{1} \prec_{L} p$ and $r \prec_{L} u_{3}$ imply the second half of (4.5).

Finally, (2.4) and (4.5) give that $\operatorname{con}\left(q, u_{2}\right)=\operatorname{con}\left(u_{1}, p\right)=\operatorname{con}\left(r, u_{3}\right)$. Since (4.4) allows us to replace $q$ and $r$ by $u_{2}^{-}$and $u_{3}^{-}$, respectively, we obtain that $\operatorname{con}\left(u_{2}^{-}, u_{2}\right)=\operatorname{con}\left(u_{3}^{-}, u_{3}\right)$. But $u_{2}$ and $u_{3}$ are distinct elements of $\mathrm{J}(L)$ by (4.4), whereby (4.1) and Lemma 4.1(ii) imply that $L$ has few congruences, as required. This completes the proof of Lemma 4.2.

We still need another lemma. 
Lemma 4.3. Let $K:=E_{0} \in \mathcal{L}_{K R}$, see on the left in Figure 2. If $K$ is a subposet of a finite lattice $L$, then $L$ has few congruences.

Proof. This proof shows a lot of similarities with the earlier one. In particular, the same convention applies for the diagram of $L$ in Figure 2 and, again, there can be several elements of $L$ not indicated in the diagram. We have already noted that (4.1) holds in the present situation. Figure 2 shows how to pick $u_{1}, u_{2} \in L$; they are covers of $a \wedge_{L} b$ in $\left[a \wedge_{L} b, a\right]$ and $b \wedge_{L} c$ in $\left[b \wedge_{L} c, c\right]$, respectively. As a counterpart of (4.2) and the paragraph following it, now we obtain in the same way from (4.1) and Lemma 3.1 that the comparabilities and incomparabilities on the right of Figure 2 are correctly depicted and

$$
\left.\begin{array}{rl}
\operatorname{JRed}(L) & =\left\{p:=a \vee_{L} b=b \vee_{L} c, e \vee_{L} f, e \vee_{L} g=f \vee_{L} g\right\} \text { and } \\
\operatorname{MRed}(L) & =\left\{a \wedge_{L} b=a \wedge_{L} d, b \wedge_{L} c=d \wedge_{L} c, a \wedge_{L} c=e \wedge_{L} f\right\} .
\end{array}\right\}
$$

Neither all the equalities above, nor all similar equalities like $d \wedge_{L} g=e \wedge_{L} f$, nor all features of the figure will be used, and there can be many more elements not indicated. Using (4.7) in the same way as we used (4.2) in the proof of Lemma 4.2 and the above-mentioned correctness of Figure 2, it follows that

$$
\left.\begin{array}{l}
{\left[a \wedge_{L} b, a\right] \backslash\left\{a \wedge_{L} b\right\} \subseteq \operatorname{Irr}(L) \text { and }[a, p] \backslash\{p\} \subseteq \operatorname{Irr}(L),} \\
\text { whereby } u_{1} \in \mathrm{J}(L) \text { and }\left[u_{1}, p\right] \text { is a chain. }
\end{array}\right\}
$$

Similarly to the argument verifying (4.5) (but $p$ need not cover $b$ and we need to use that $\left[u_{1}, p\right]$ is a chain), (4.8) implies that $\left[u_{1}^{-}, u_{1}\right]=\left[a \wedge_{L} b, u_{1}\right] \nearrow[b, p]$. Since $\left\langle a, u_{1}\right\rangle$ and $\left\langle c, u_{2}\right\rangle$ play symmetric roles, we obtain that $u_{2} \in \mathrm{J}(L)$ and $\left[u_{2}^{-}, u_{2}\right]=\left[b \wedge_{L} c, u_{2}\right] \nearrow[b, p]$. Hence, $(2.4)$ gives that $\operatorname{con}\left(u_{1}^{-}, u_{1}\right)=\operatorname{con}(b, p)=$ $\operatorname{con}\left(u_{2}^{-}, u_{2}\right)$. Since $u_{1}$ and $u_{2}$ are distinct by Figure 2 and they belong to $\mathrm{J}(L)$ by (4.8) and the $\left\langle a, u_{1}\right\rangle-\left\langle c, u_{2}\right\rangle$-symmetry, (4.1) and Lemma 4.1(ii) imply that $L$ has few congruences. This completes the proof of Lemma 4.3.

Now, we are in the position to prove our theorem.

Proof of Theorem 1.1. Let $L$ be an arbitrary non-planar finite lattice; it suffices to show that $L$ has few congruences. By Proposition 2.1, there is a lattice $K$ in Kelly and Rival's list $\mathcal{L}_{\mathrm{KR}}$ such that $K$ is a subposet of $L$ or the dual $L^{\text {dual }}$ of $L$. Since $\operatorname{Con}\left(L^{\text {dual }}\right)=\operatorname{Con}(L)$ and $L^{\text {dual }}$ is non-planar either, we can assume that $K$ is a subposet of $L$. A quick glance at the lattices of $\mathcal{L}_{\mathrm{KR}}$, see their diagrams in Kelly and Rival [9], shows that if $K \in \mathcal{L}_{\mathrm{KR}} \backslash\left\{E_{0}, F_{0}\right\}$, then $|\operatorname{JRed}(K)| \geq 4$ or $|\operatorname{MRed}(K)| \geq 4$. Hence, if $K \in \mathcal{L}_{\mathrm{KR}} \backslash\left\{E_{0}, F_{0}\right\}$, then Lemma 4.1(i) implies that $L$ has few congruences, as required. If $K \in\left\{E_{0}, F_{0}\right\}$, then the same conclusion is obtained by Lemmas 4.2 and 4.3. This completes the proof of Theorem 1.1. 


\section{Acknowledgements}

Open access funding provided by University of Szeged (SZTE). The referee's historical comment calling the author's attention to Crawley and Dilworth [2] and to Dilworth [4] is highly appreciated.

Open Access. This article is distributed under the terms of the Creative Commons Attribution 4.0 International License (http://creativecommons.org/licenses/ by/4.0/), which permits unrestricted use, distribution, and reproduction in any medium, provided you give appropriate credit to the original author(s) and the source, provide a link to the Creative Commons license, and indicate if changes were made.

Publisher's Note Springer Nature remains neutral with regard to jurisdictional claims in published maps and institutional affiliations.

\section{References}

[1] Adaricheva, K., Czédli, G.: Note on the description of join-distributive lattices by permutations. Algebra Univ. 72, 155-162 (2014)

[2] Crawley, P., Dilworth, R.P.: Algebraic Theory of Lattices. Prentice, Englewood Cliffs (1973)

[3] Czédli, G.: A note on finite lattices with many congruences. Acta Universitatis Matthiae Belii Ser. Math. Online, pp. 22-28. http://actamath.savbb.sk/pdf/ oacta2018003.pdf (2018)

[4] Dilworth, R.P.: The structure of relatively complemented lattices. Ann. Math. 51, 348-359 (1950)

[5] Freese, R.: Computing congruence lattices of finite lattices. Proc. Am. Math. Soc. 125, 3457-3463 (1997)

[6] Freese, R., Ježek, J., Nation, J.B.: Free lattices. In: Mathematical Surveys and Monographs, vol. 42. American Mathematical Society, Providence (1995)

[7] Grätzer, G.: Lattice Theory: Foundation. Birkhäuser, Basel (2011)

[8] Grätzer, G.: Congruences and prime-perspectivities in finite lattices. Algebra Univ. 74, 351-359 (2015)

[9] Kelly, D., Rival, I.: Planar lattices. Can. J. Math. 27, 636-665 (1975)

[10] Kulin, J., Mureşan, C.: Some extremal values of the number of congruences of a finite lattice. arxiv:1801.05282 (2018)

[11] Mureşan, C.: Cancelling congruences of lattices while keeping their filters and ideals. arxiv:1710.10183 (2017)

[12] Nation, J.B.: Notes on Lattice Theory. http://www.math.hawaii.edu/ jb/books. html 
[13] Rival, I.: Lattices with doubly irreducible elements. Can. Math. Bull. 17, 91-95 (1974)

Gábor Czédli

Bolyai Institute

University of Szeged

Szeged 6720

Hungary

e-mail: czedli@math.u-szeged.hu

URL: http://www.math.u-szeged.hu/ czedli/

Received: 22 July 2018.

Accepted: 7 February 2019. 